\title{
EL CONSUMO RESPONSABLE DE LOS RECURSOS NATURALES COMO PUNTO DE PARTIDA PARA UN DESARROLLO SUSTENTABLE: UNA APROXIMACIÓN CRÍTICA.
}

\author{
José Alonzo Sahui Maldonado*
}

\begin{abstract}
Sahui-Maldonado J.A. El consumo responsable de los recursos naturales como punto de partida para un desarrollo sustentable: Una aproximación crítica. Hitos de Ciencias Económico Administrativas 2012;18 (51):63-72.
\end{abstract}

\section{RESUMEN}

Este trabajo parte de la idea de que en la mayoría de las sociedades más desarrolladas existe un consumo excesivo de recursos naturales, así como prácticas de consumo que son perjudiciales para el medio ambiente. Por lo tanto, se hace un breve análisis de cuatro de estos recursos (energía, minerales, agua y tierra cultivable), respectivamente. Se concluye que es necesario privilegiar el concepto de sustentabilidad en el diseño de las políticas públicas, para lo cual se propone como una alternativa viable, la teoría del desarrollo a escala humana de Manfred A. Max-Neef.
Sahui-Maldonado J.A. The responsible use of natural resources as a basis for sustainable development: $A$ critical approach. Hitos de Ciencias Económico Administrativas 2012;18 (51):63-72.

\section{ABSTRACT}

This work is based on the idea that in most of the developed societies there is an excessive consumption of natural resources, as well as consumption practices that are harmful to the environment. Therefore, in this paper a brief analysis of four of these resources is done -energy, minerals, water and arable landrespectively. It is concluded that it is necessary to privilege the concept of sustainability in the design of public policies, and so, as a viable alternative, the Theory of Human Scale Development by Manfred A. Max-Neef is proposed.

Palabras clave: Consumo.Desarrollo. Recursos

Key words: Consumption. Development. Natural. naturales. Resources.

DIRECCIÓN PARA RECIBIR CORRESPONDENCIA: Correo electrónico josesahui@yahoo.com.mx

uando se habla de consumo y desarrollo, se alude a dos términos que en el ámbito de las Ciencias Sociales son de uso cotidiano que, paradójicamente, la misma familiaridad genera problemas a la hora de establecerlos como objetos de estudio. Por ejemplo, el caso del consumo; se encuentra el hecho de que éste no es una actividad que solamente se limita a la satisfacción de las necesidades básicas de una persona. En el consumo, hay de manera implícita una serie de valores simbólicos de carácter sociocultural que hacen de esta actividad -el consumir- algo bastante complejo.
Por otro lado, con el concepto de desarrollo las cosas son demasiado parecidas, debidoa a que, mientras que en términos biológicos, éste se define como el proceso de crecimiento de un organismo hasta alcanzar su madurez, en las Ciencias Sociales generalmente sirve para designar el proceso de cambio por medio del cual las sociedades de los países no industrializados van adquiriendo las características de las sociedades industrializadas, motivo por el cual, éstas últimas se constituyen en el parámetro a través del cual se mide el nivel de desarrollo alcanzado por otras sociedades, sea éste del tipo que fuere: económico, social, sustentable o humano.

\footnotetext{
* Doctor. Profesor-Investigador. Facultad de Contaduría y Administración. Universidad Autónoma de Campeche.
}

Fecha de recibido: 15 de enero de 2012 Fecha de aceptación: 3 de marzo de 2012. 
Para tal efecto, el presente trabajo busca realizar una aproximación de ambos conceptos. De entrada se trata de hacerlos más específicos al añadirle a cada término un adjetivo (responsable y sustentable), respectivamente. Con esto se pretende construir un espacio de análisis para establecer una serie de reflexiones en torno a la siguiente idea: que el desarrollo sustentable, mismo que se define como el desarrollo que satisface las necesidades de la generación presente, sin comprometer la capacidad de las generaciones futuras para satisfacer sus propias necesidades, seguirá siendo una falacia mientras no se practique un consumo responsable. Situación que, desafortunadamente, la sociedad actual está todavía bastante lejos de practicar.

\section{El consumismo: una paradoja del desarrollo}

En su teoría del desarrollo, Walt Whitman Rostow, señala la existencia de cinco etapas por las que atraviesan todas las sociedades (Salguero Cubides, 2006). Así, puede observarse cómo las sociedades van «evolucionando» desde una primera etapa de autosuficiencia en donde prácticamente la totalidad de la producción está destinada al consumo de los productos; hasta una última etapa, caracterizada por el consumo a gran escala. Esta etapa, que históricamente coincidió con la llamada segunda revolución industrial [1870-1914], se identifica a su vez, con la aparición de los sistemas de administración y mercadotecnia que en la actualidad utilizan prácticamente todas las empresas.

En este sentido, la hipótesis de Rostow acerca de que el fenómeno consumista se da de manera paralela al desarrollo de las sociedades, es digna de tomarse en cuenta, sobre todo cuando el concepto de desarrollo se traduce -en la mayoría de los países- en un aumento indiscriminado en el número de opciones de bienes y servicios que satisfacen nuestras necesidades y deseos. Razón por la que en gran medida, muchos teóricos sociales señalan que estamos viviendo en una sociedad de consumo, siendo el consumismo el espíritu que permea en dicha sociedad.

Ahora bien, según el Diccionario de la Real Academia (2008), el consumismo puede definirse como «la tendencia inmoderada a adquirir, gastar o consumir bienes, no siempre necesarios». No obstante, el problema de la definición anterior es ¿cómo distinguir lo necesario de lo que no lo es?...¿cómo distinguir el consumo «normal» del consumo «excesivo»?. Tal parece que cuando uno aborda el problema del consumismo es muy fácil caer en la tentación de criticar solo lo que compran los demás, y no hacer un análisis más profundo de las causas que motivan a las personas a este aparente consumo excesivo u ostentoso, de acuerdo con la terminología utilizada por Thorstein Veblen (2004) en su Teoría de la Clase Ociosa.

Por otra parte, es indudable que algunas prácticas de consumo han comenzado a dañar seriamente los sistemas naturales de los que dependemos, dificultando de esta forma que los habitantes de los países pobres puedan satisfacer sus necesidades básicas. Por ejemplo, según el Informe del World Watch Institute sobre «La situación del mundo 2004»; mientras que los consumidores de América del Norte y Europa Occidental gastan 35,000 millones de dólares al año en agua envasada, en el mundo existen 1,100 millones de personas que carecen de acceso al agua potable. De igual forma, el citado informe señala que los más ricos del mundo utilizan en promedio 25 veces más energía que los pobres, así como Estados Unidos, con solo un $4.5 \%$ de la población mundial, libera un $25 \%$ de todas las emisiones de dióxido de carbono (citado por Elizalde Hevia, 2007).

Asimismo, según ciertas estimaciones planteadas en el Informe de Desarrollo Humano 1998, preparado para el Programa de Naciones Unidas para el Desarrollo (PNUD, 1998), se indica que los 225 habitantes más ricos del mundo tienen una riqueza combinada igual al ingreso anual del $47 \%$ más pobre de la población mundial -aproximadamente 2,500 millones de habitantes-.

Todo lo anterior, se hace más evidente, se comparan los costos de ciertos servicios básicos de los países en desarrollo en relación con el consumo de ciertos bienes y servicios de los países desarrollados (ver tabla 1).

No obstante, el aspecto más alarmante de la situación actual no estriba tanto en las condiciones medioambientales per se, sino en el comportamiento y la actitud con que los seres humanos se enfrentan al mundo. Esto es quizás lo que ha llevado a algunos 


\section{TABLA I. COMPARATIVO DE COSTOS DE SERVICIOS BÁSICOS VS GASTO EN CONSUMO.}

\begin{tabular}{|c|c|}
\hline $\begin{array}{l}\text { Estimación del costo anual adicional de } \\
\text { lograr el acceso universal a servicios } \\
\text { básicos en todos los países en desarrollo. }\end{array}$ & $\begin{array}{l}\text { Gasto en consumo de diversos bienes y } \\
\text { servicios en países desarrollados. }\end{array}$ \\
\hline $\begin{array}{l}\text { Enseñanza básica para todos }=6 \text { mil millones } \\
\text { de dólares. }\end{array}$ & $\begin{array}{l}\text { Cosméticos en los Estados Unidos }=8 \mathrm{mil} \\
\text { millones de dólares. }\end{array}$ \\
\hline $\begin{array}{l}\text { Agua y saneamiento para todos }=9 \text { mil millones } \\
\text { de dólares. }\end{array}$ & $\begin{array}{l}\text { Helados en Europa= } 11 \text { mil millones de } \\
\text { dólares. }\end{array}$ \\
\hline $\begin{array}{l}\text { Salud reproductiva para todas las mujeres }=12 \\
\text { mil millones de dólares. }\end{array}$ & $\begin{array}{l}\text { Perfumes en Europa y los Estados Unidos= } \\
12 \text { mil millones de dólares. }\end{array}$ \\
\hline \multirow[t]{2}{*}{$\begin{array}{l}\text { Salud y nutrición básicas }=13 \text { mil millones de } \\
\text { dólares. }\end{array}$} & $\begin{array}{l}\text { Alimento para animales domésticos en } \\
\text { Europa y los Estados Unidos }=17 \text { mil } \\
\text { millones de dólares. }\end{array}$ \\
\hline & $\begin{array}{l}\text { *Otros datos interesantes.- } \\
\text { Cigarrillos en Europa }=50 \text { mil millones de } \\
\text { dólares } \\
\text { Bebidas alcohólicas }=105 \text { mil millones de } \\
\text { dólares } \\
\text { Gasto militar en el mundo= } 780 \text { mil millones } \\
\text { de dólares }\end{array}$ \\
\hline
\end{tabular}

Fuente: Elizalde Hevia (2007).

autores, como Joseph Wood Krutch, a señalar que «aún cuando la especie biológica homo sapiens ha continuado prosperando, la humanidad comenzó a degenerar en algún momento de finales del siglo XIX, cuando los deseos de la sociedad de consumo tomaron precedencia sobre las aspiraciones culturales y espirituales» (citado por René Dubos, 1985,43). A efecto de dejar evidencia de lo anterior, a continuación se presenta un breve diagnóstico del consumo excesivo de algunos recursos naturales.

\section{Diagnóstico acerca del consumo excesivo de cuatro recursos naturales}

\section{Energía}

Si en algo se diferencia nuestra especie del resto de las especies animales -además de la posesión de un lenguaje articulado capaz de transmitir conocimientos de una generación a otra- es en el uso de la energía; y no es que las otras especies no la utilicen, el hecho es que los seres humanos somos los únicos que hemos aprendido a usar, como fuentes de energía, elementos ajenos a nuestra constitución biológica, ya sea para movernos, comunicarnos o defendernos.

Por ejemplo, el caso de la invención de la rueda o el descubrimiento del fuego. Es indudable, que este último se convirtió en un recurso que permitió a nuestros antepasados convertirse en una especie capaz de someter a otras especies, modificar su entorno y, por consiguiente, expandir su dominio a otras regiones. El problema, en este sentido, es que estamos llegando-en lo que respecta al consumo de energía-, y dadas las condiciones medioambientales actuales, a padecer lo que René Dubos bautizó en su libro Celebraciones de la vida (1985) como el síndrome de Beauvais".

${ }^{1}$ René Dubos (1985), bautizó como el síndrome de Beauvais a la tendencia de las civilizaciones a desarrollar hasta el absurdo algunas de las actitudes prácticas y técnicas a las que debieron su éxito inicial. 
Para demostrar lo anterior, (ver figura 1) donde se señala el aumento en el consumo energético a través de diferentes etapas de la historia.

\section{Sociedad Kilocalorías por persona y día}

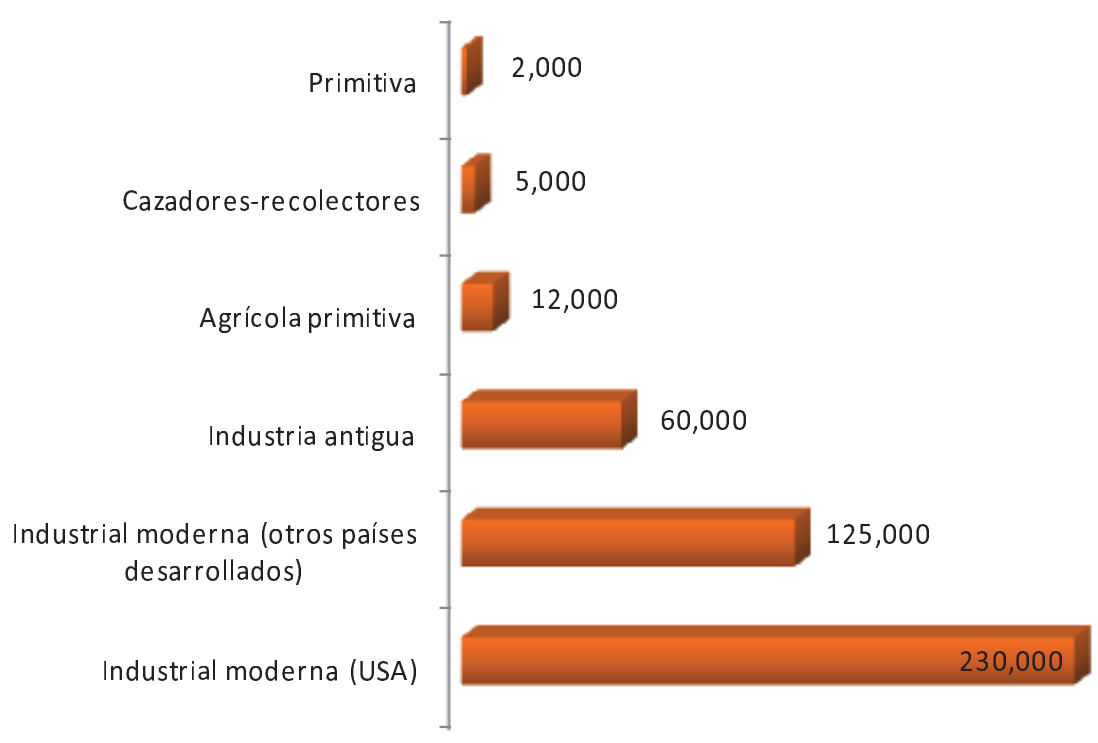

FIGURA 1. CONSUMO DE ENERGÍA EN DIFERENTES ETAPAS DE LA SOCIEDAD.

Fuente: Elizalde Hevia (2007).

Un último punto digno de tomar en cuenta en este apartado, es el caso del petróleo, ya que según algunas estimaciones «cada minuto se queman 60 millones de barriles de petróleo, con un gran gasto, liberando a la atmósfera óxidos de azufre y nitrógeno, lo que provoca lluvia ácida, y bióxido de carbono que amenaza con generar cambios climáticos globales» (Meadows, 1988, 60). Esto, aunado al hecho de que cerca del $80 \%$ de la energía comercial del mundo proviene de los llamados combustibles fósiles (petróleo, gas y carbón).

\section{Minerales}

En la actualidad, los seres humanos extraen una gran cantidad de minerales de la tierra y se eliminan cerca de la misma cantidad en la forma de desechos sólidos. Esto significa que las reservas minerales a nivel mundial han permanecido más o menos constantes, al menos en lo que respecta al binomio extracción/consumo. No obstante, el problema radica en que los yacimientos de donde se obtiene la mayoría de los minerales se encuentran cada vez más dispersos, lo cual incrementa considerablemente los costos en lo que respecta a capital, trabajo y energía, así como el daño ecológico ocasionado por la explotación de dichos yacimientos.
Así pues, y «dado que los depósitos minerales de alta concentración son muy limitados, se están agotando y cada vez son más difíciles de encontrar, es obvio que tenemos que hacer conciencia del uso racional de los minerales y la necesidad de reciclarlos tanto como sea posible» (Meadows, 1988, 63).

Por otra parte, el consumo de los minerales en los países desarrollados-a pesar del uso cada vez más frecuente de los procesos de reciclaje- sigue estando muy por arriba del consumo de los países en vías de desarrollo (ver tabla II).

\section{Agua}

A pesar de que en el mundo existen provisiones de agua suficientes para cerca del doble de la población actual (Meadows, 1988), debido a los patrones de consumo en la actualidad, así como a los diversos usos -agricultura, industria y consumo doméstico-, que le damos a este recurso de manera excesiva, el «problema del agua» es más bien resultado de la contaminación que de la escasez.

Sin embargo, es importante destacar que el consumo de agua a nivel mundial se ha triplicado desde 1950 hasta el año 2006, sobrepasando los 4.300 kilómetros cúbicos/año ${ }^{2}$, cifra equivalente al $30 \%$ de la dotación mundial de agua renovable. De igual forma, cabe señalar que según información contenida en el informe presentado por la UNICEF con motivo del Día Mundial del Agua,

2 Un kilómetro cúbico equivale a 1,000 hectómetros cúbicos; es decir, a mil millones de metros cúbicos. 
TABLA II. CONSUMO DE METALES Y MINERALES EN EL 2000.

Metal ylo Mineral

(Miles de toneladas)

\begin{tabular}{|c|c|c|c|c|c|c|c|}
\hline & $\begin{array}{l}\text { América } \\
\text { del Norte }\end{array}$ & $\begin{array}{l}\text { América } \\
\text { del Sur }\end{array}$ & Europa & ExURSS & Asia & África & Otro \\
\hline Aluminio & 7.291 & 823 & 6.632 & 612 & 8.819 & 294 & 421 \\
\hline Plomo & 1.924 & 212 & 1.854 & 179 & 1.866 & 118 & 47 \\
\hline Zinc & 1.714 & 352 & 2.572 & 280 & 3.563 & 162 & 240 \\
\hline Cobre & 3.649 & 534 & 4.551 & 270 & 5.868 & 116 & 176 \\
\hline Níquel & 165 & 24 & 416 & 25 & 449 & 31 & 2 \\
\hline $\begin{array}{l}\text { Acero (millones } \\
\text { de toneladas) }\end{array}$ & 170 & 33 & 206 & 25 & 377 & 18 & 9 \\
\hline Oro (toneladas) & 306 & 83 & 906 & 42 & 2.423 & 179 & 7 \\
\hline $\begin{array}{l}\text { Carbón (millones } \\
\text { de toneladas de } \\
\text { equivalente } \\
\text { petróleo) }\end{array}$ & 613 & 37 & 241 & 197 & 767 & 123 & 158 \\
\hline Fosfato mineral & 44.580 & 6.298 & 11.008 & 8.965 & 43.210 & 23.087 & 2.718 \\
\hline
\end{tabular}

Fuente: CRU Internacional, 2001.
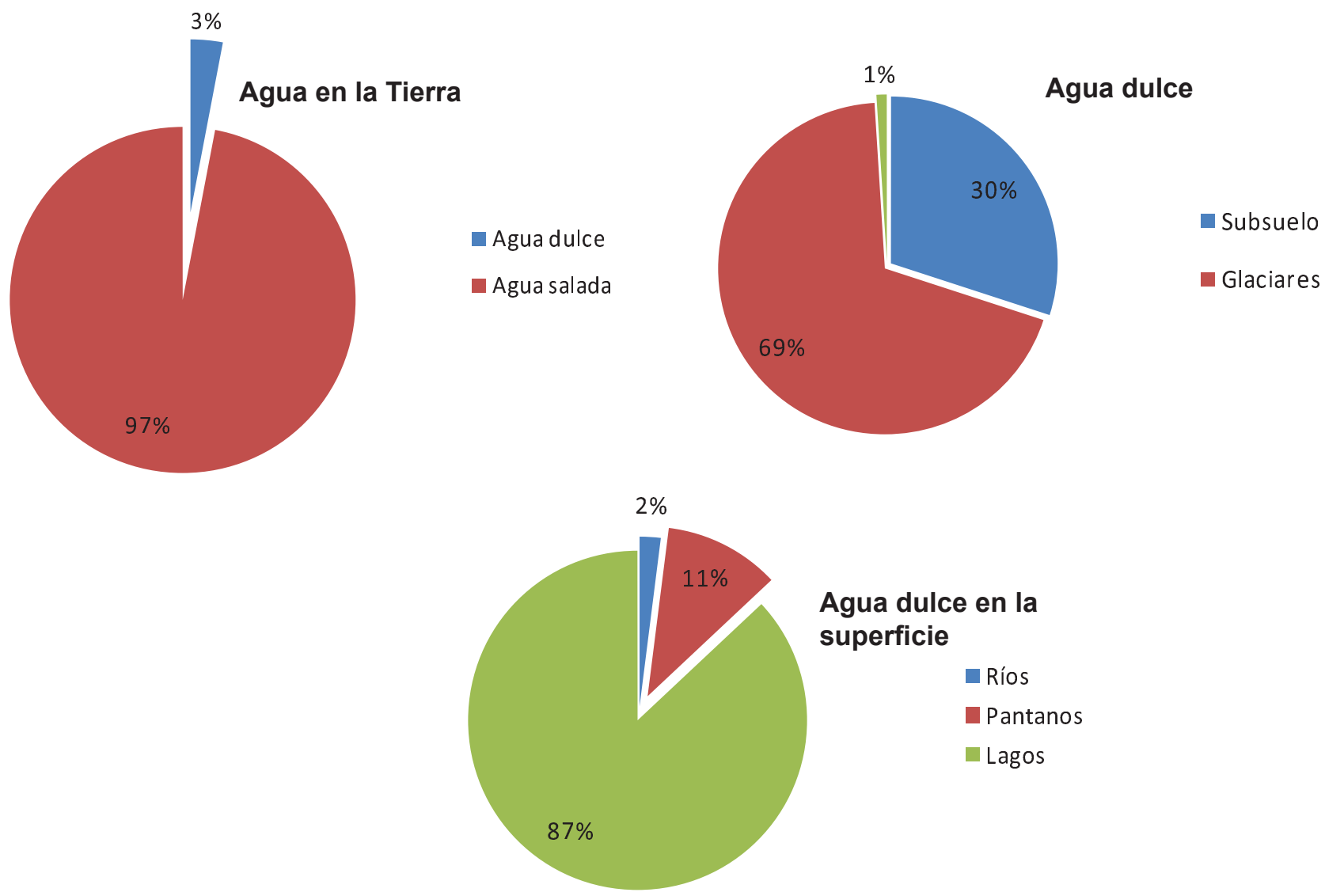

FIGURA 2. DISTRIBUCIÓN DEL AGUA EN LA TIERRA

Fuente: World Resources Institute (citado por el INE, 2008). 
TABLA III. DEMANDA Y OFERTA ECOLÓGICA EN PAÍSES SELECCIONADOS, 2003.

\begin{tabular}{lcccc} 
& $\begin{array}{l}\text { Huella Ecológica } \\
\text { total (millones de } \\
\text { hag) }\end{array}$ & $\begin{array}{l}\text { Huella Ecológica } \\
\text { percápita } \\
\text { (hag/persona) }\end{array}$ & $\begin{array}{l}\text { Biocapacidad } \\
\text { (hag/persona) }\end{array}$ & $\begin{array}{l}\text { Reservaldéficit } \\
\text { (-)ecológico } \\
\text { (hag/persona) }\end{array}$ \\
\hline $\begin{array}{l}\text { Mundo } \\
\text { Estados Unidos de }\end{array}$ & 14.144 & 2,2 & 1,8 & $-0,4$ \\
América & 2819 & 9,6 & 4,7 & $-4,8$ \\
China & 2152 & 1,6 & 0,8 & $-0,9$ \\
India & 802 & 0,8 & 0,4 & $-0,4$ \\
Federación de Rusia & 631 & 4,4 & 6,9 & 2,5 \\
Japón & 556 & 4,4 & 0,7 & $-3,6$ \\
Brasil & 383 & 2,1 & 9,9 & 7,8 \\
Alemania & 375 & 4,5 & 1,7 & $-2,8$ \\
Francia & 339 & 5,6 & 3,0 & $-2,6$ \\
Reino Unido & 333 & 5,6 & 1,6 & $-4,0$ \\
México & 265 & 2,6 & 1,7 & $-0,9$ \\
Canadá & 240 & 7,6 & 14,5 & 6,9 \\
Italia & 239 & 4,2 & 1,0 & $-3,1$ \\
\hline
\end{tabular}

Fuente: Global Footprint network, 2006.

«al menos el $20 \%$ de la población mundial -que se estima ese año en 6.658 millones de habitantes- carece de acceso al agua potable y que el $42 \%$ no tiene un grifo que abrir para su aseo personal y no dispone de servicios adecuados de alcantarillado y depuración de las aguas residuales» (citado por el Instituto Nacional de Estadística, 2008).

En este sentido, y dada la distribución desigual del agua a nivel mundial, es importante establecer estrategias que permitan un mejor manejo de este recurso que es esencial para la vida.

\section{Tierra cultivable}

A pesar de que "cada día se pierden por erosión 57 millones de toneladas de suelo, suficientes para cubrir 23,000 hectáreas con una capa de 20 centímetros de profundidad» (Meadows, 1988, 54), la cantidad de alimentos que se producen actualmente a nivel mundial se ha incrementado de manera más rápida que la población durante los últimos 30 años. En gran medida, este incremento de la producción de alimentos se debe a la utilización de tecnologías agrícolas más productivas que, desafortunadamente, no son sustentables. Así, el $40 \%$ de la tierra cultivada en los Estados Unidos se está erosionando a un ritmo acelerado, debido principalmente a sus prácticas de cultivo intensivo ${ }^{3}$.

Por otro lado, en muchos países no desarrollados, en donde la productividad agrícola es baja, la erosión es también un problema serio, aunque por razones diferentes; como son: la sobrepoblación humana y ganadera, así como la deforestación. En relación con este punto, cabe señalar que la pérdida anual de tierra forestal provoca -como efectos secundarios- erosión del suelo, sequías e inundaciones y la desaparición de diversas especies animales y vegetales.

En este sentido, y dado que la deforestación y pérdida de la tierra cultivable es uno de los problemas más serios en lo que se refiere al uso y consumo que se

\footnotetext{
${ }^{3}$ Por ejemplo, «el sistema agrario norteamericano, reconocido por su enorme eficiencia, es un sistema notablemente ineficiente si se le mide en términos de la cantidad de energía consumida para producir una cantidad determinada de kilo/calorías. No obstante, si se le mide en términos monetarios, genera beneficios enormes» (Max-Neef, et. al., 1998). De igual forma, Dubos (1985) señala que «el $34 \%$ del aumento de la producción de alimentos en Estados Unidos entre 1951 y 1956 se acompañó de un 146\% de aumento en el uso de nitratos y $300 \%$ en el de plaguicidas» (p. 292). [Como puede observarse con estos dos ejemplos, la tecnología moderna, no siempre es necesariamente la más conveniente].
} 
hacen de los recursos naturales, el desarrollo de conceptos como el de huella ecológica, mismo que busca determinar el peso o carga ambiental de una persona -o una sociedad- en función de su nivel de consumo, se constituye en un indicador muy eficaz para poder estimar cuanto utilizan las personas de la capacidad productiva del planeta. Así pues, en la siguiente tabla se presentan algunos datos reveladores:

\section{¿Cómo lograr un desarrollo sustentable?}

Como se puede apreciar en el diagnóstico anterior, es claro que el consumo excesivo de los recursos naturales se constituye en una grave amenaza. Algunos consideran que el materialismo y la industrialización de la sociedad han sido los principales causantes del deterioro de los recursos anteriormente descritos; no obstante, como atinadamente señala Dubos (1985); «La tierra ha sido siempre escasa en recursos naturales. Las materias se convierten en recursos sólo después de haberlas extraído de los materiales brutos que las contienen y haberlas manipulado con algún propósito deliberado para que el hombre las utilice» (p. 272).

Por tanto, el problema no estriba tanto en los procesos de transformación de los recursos naturales, sino en la forma desmedida en que los consumimos, en ese consumo descuidado y despilfarrador de una sociedad que puede obtener los bienes que necesita en exceso y sin prácticamente ningún esfuerzo, y esto se hace evidente cuando analizamos la información comparativa del consumo de diversos recursos en los países desarrollados, con respecto a los países en vías de desarrollo.

En este sentido, la teoría del desarrollo a escala humana de Manfred A. Max-Neef (Max Neef, et. al., 1983), se constituye en una propuesta digna de tomarse en cuenta. Esta teoría parte de la idea de que el desarrollo debe orientarse preferentemente hacia la satisfacción de las necesidades básicas ya que «las pautas de consumo que el mundo rico exporta e impone al mundo en desarrollo somete a este último a relaciones de intercambio que agudizan su dependencia, perpetúan sus desequilibrios internos y amenazan su identidad cultural».
De igual forma, y en gran medida influida por los autores antes citados, Larraín (2002) propone como un marco ético y político para el desarrollo sustentable el concepto de línea de dignidad ${ }^{4}$, mismo que «pretende conciliar los objetivos de la sustentabilidad ambiental con los objetivos distributivos de la equidad social y la democracia participativa [...] Pretende establecer los parámetros para un nuevo indicador social, que eleva el nivel de satisfacción de necesidades establecidas en la «línea de pobreza» a una nueva línea base, concebida como de dignidad humana y establecida bajo un enfoque de necesidades humanas ampliadas» (p. 1).

En este mismo orden de ideas, la autora plantea un concepto de desarrollo sustentable, mismo que se anexa a continuación, y que se considera una propuesta adecuada dado que involucra aspectos ambientales, sociales y políticos (Larraín, 2002: 25):

El concepto de sustentabilidad: integrando la dimensión social, ambiental y política del desarrollo

1. Sustentabilidad ambiental: El desafío de mantener la vida en el planeta

- Protección de los sistemas biofísicos que permiten mantener las funciones del planeta.

- Uso sustentable de los ecosistemas y la naturaleza.

- Satisfacer las necesidades de los seres humanos y las demás especies en el presente $y$ en el futuro (futuras generaciones).

- Instrumentos y regulaciones legales y económicas para asegurar la sustentabilidad ambiental.

2. Sustentabilidad social: El desafío de la equidad Superación de la pobreza.

Mejorar la distribución del ingreso o de la riqueza.

- Satisfacción de las necesidades humanas (con base al ejercicio de derechos y no solo de

${ }^{1}$ En torno a esta idea, algunos autores como Elizalde Hevia (2007), han manifestado que hay indignidad no solamente en el subconsumo de los pobres, sino también en el sobreconsumo de los ricos. 
aquellas restringidas al gasto social tradicional).

Equidad entre géneros y culturas.

- Equidad entre regiones a nivel nacional.

- Equidad entre sociedades del norte y del sur.

3. Sustentabilidad política: El desafío de la democracia participativa y la profundización democrática

- Ejercicio de derechos humanos.

- Participación ciudadana y gobernabilidad democrática.

- Participación democrática en los sistemas políticos.

- Descentralización territorial y de la toma de decisiones.

- Ejercicio de derecho y participación de los pueblos indígenas.

- Ejercicio de derechos y participación de las mujeres y los jóvenes.

- Reglas legales e instituciones para la sustentabilidad política.
En este sentido, para la aplicación del concepto de sustentabilidad en el diseño de las políticas públicas dirigido a las empresas comerciales, industriales y de servicios, se podría seguir en el proceso presentado en la Tabla IV.

\section{Conclusión}

Ningún país, desarrollado o en vías de desarrollo, ha logrado impulsar un sistema de administración de sus recursos naturales totalmente eficiente. Sin embargo, el manejo adecuado de los mismos, es una oportunidad única para establecer políticas de cooperación y solidaridad internacionales, debido a que las lecciones que se aprenden en una región -sean éstas positivas o negativas- pueden y deben ser aprovechadas en otras regiones del planeta.

Por otro lado, es indudable que si todos consumiéramos de manera responsable y moderada, la sociedad funcionaría en ese mismo sentido. No obstante, vivimos en una sociedad que todavía no aprende a distinguir las necesidades verdaderas de las necesidades falsas ${ }^{5}$.

\section{TABLA IV. FASES EN EL CAMINO HACIA LA SUSTENTABILIDAD POR PARTE DE LAS EMPRESAS}

\section{Fases}

1. Reconocer oportunidad que existe en el cumplimiento de las normas.

2. Hacer sustentable la cadena de valor.

3. Diseñar productos $y$ servicios sustentables.

4. Desarrollar nuevos modelos de negocios.
5. Crear plataformas para las prácticas venideras.

Estrategias principales para desarrollarla

\begin{abstract}
Asegurándose que el cumplimiento de las normas ambientales se convierta en una oportunidad para la innovación, desarrollando la habilidad de trabajar con otras compañías, incluso rivales, para implementar soluciones creativas.
\end{abstract}

Aumentando la eficiencia en toda la cadena de valor, rediseñando las operaciones a fin de que las empresas usen menos energía en sus procesos de transformación, produzcan menos emisiones contaminantes y generen menos desperdicios.

Desarrollando of ertas sustentables o rediseñando las existentes para que sean amigables con el entorno.

Encontrando nuevas formas de entregar y capturar valor, las que cambiarán los principios fundamentales de la competencia.

Cuestionando desde la perspectiva de la sustentabilidad la lógica dominante que subyace en los negocios en la actualidad.

Fuente: Elaboración propia con base en el artículo «¿Por qué la sustentabilidad es hoy el impulsor clave de la innovación?» (Nidumolu, et. al., 2010). 
Y mientras se siga así, el consumo excesivo de los bienes que la sociedad produce, seguirá creciendo de manera exponencial.

De igual forma, y en lo que respecta al ámbito empresarial, es importante también que en los negocios el concepto de sustentabilidad deje de considerarse como una carga, que se refleja en el incremento de sus costos y se comience a ver como un medio que impulse la innovación. Desde luego, para lograr lo anterior, se necesita que se replanteen muchas de las formas en que tradicionalmente se han estado llevando los negocios. Sin embargo, el auge de conceptos, tales como: mercadotecnia social, responsabilidad social corporativa y ética empresarial, entre otros; parece que apuntan en esta dirección.

En este sentido, las conclusiones del presente trabajo indican que sí es posible consumir con responsabilidad y haciendo de nuestra relación con las fuerzas del mercado un espacio de reflexión y organización ciudadana. Por consiguiente, las recomendaciones a manera de conclusión son las siguientes:

- Es necesario que la sociedad civil busque mecanismos y estrategias para empoderar a la gente; pues, aunque las organizaciones de consumidores están participando más activamente, frente a los gobiernos y a los mercados, el consumo -a final de cuentas- es una decisión personal.

- Es necesario que los gobiernos participen más activamente defendiendo los derechos de las personas frente a los intereses de las grandes empresas. Las fallas actuales del sistema capitalista han terminado por demostrar que ninguno de los extremos es recomendable: ni el capitalismo voraz, ni el socialismo estatista.

- Es necesario que la sociedad civil le exija al gobierno políticas económicas y sociales, diseñadas para servir a las personas y al medio ambiente en el que se desarrollan.

\footnotetext{
${ }^{5}$ «Marcuse distingue dos tipos de necesidades -verdaderas y falsasque los individuos intentan satisfacer al consumir. Verdaderas son las necesidades vitales, como alimentación, vestido o vivienda; falsas son las que determinadas fuerzas sociales imponen a los individuos reprimiéndoles, y que no hacen sino perpetuar la agresividad, la miseria y la injusticia» (Cortina, 2002).
}

- Finalmente, es necesario desarrollar una verdadera cultura de consumo que tenga como característica principal la responsabilidad social; una cultura que transforme a los consumidores en auténticos ciudadanos.

\section{REFERENCIAS}

Cortina, A. (2002). Por una ética del consumo. La ciudadanía del consumidor en un mundo global, España: Taurus.

Commodities Research Unit [CRU] (2001) Tomado de http://crugroup.com

Diccionario de la Real Academia Española de la Lengua (2008). Tomado de www.rae.es

Dubos, R. (1985). Celebraciones de la vida. México. Fondo de Cultura Económica.

Elizalde Hevia, A. (2007). La insuficiencia de lo suficiente: sobre cegueras yadicciones civilizatorias [documento en formato electrónico], Chile. Universidad Bolivariana.

Global Footprint Network. (2006). Informe Planeta Vivo 2006. Colombia:WWF.

Instituto Nacional de Estadística. (2008). Boletín Informativo del Instituto Nacional de Estadística (INE). Tomado de www.ine.es

Larraín, S. (2002), La línea de dignidad como indicador de sustentabilidad socioambiental: avances desde el concepto de vida mínima hacia el concepto de vida digna», en Polis: Revista Académica. Chile: Universidad Bolivariana.

Max-Neef, M., et. al. (1998). Desarrollo a escala humana: Conceptos, aplicaciones y algunas reflexiones. España. Icaria.

Meadows, D. (1988). Un breve informe sobre el estado de los recursos mundiales, en Estudios del siglo 21, Barney, G. y Alonso, A. [compiladores]. México. Centro de Estudios Prospectivos/LIMUSA.

Nidumolu, R., et. al. (2010). ¿Por qué la sustentabilidad es hoy el impulsor clave de la innovación? en Harvard Business Review América Latina, Agosto de 2010, México.

Programa de Naciones Unidas para el Desarrollo [PNUD]. (1998). Informe de Desarrollo Humano, Tomado de www.pnud.org

Salguero Cubides, J. (2006). Enfoques sobre algunas teorías referentes al desarrollo regional», en Sociedad Geográfica de Colombia. Tomado de www.sogecol.edu.co

Veblen, T. (2004). Teoría de la clase ociosa. México. Fondo de Cultura Económica. 